\title{
Gravity-driven granular flow in a silo: Characterizing local forces and rearrangements
}

\author{
Emma Thackray ${ }^{1, \star}$ and Kerstin Nordstrom ${ }^{1, \star \star}$ \\ ${ }^{1}$ Mount Holyoke College
}

\begin{abstract}
While the gravity-driven flow of a granular material in a silo geometry can be modeled by the Beverloo equation, the mesoscale-level particle rearrangements and interactions that drive this flow are not wellunderstood. We have constructed a quasi-two-dimensional system of bidisperse, millimeter-scale disks with photoelastic properties that make force networks within the material visible. The system is contained in an acrylic box with an adjustable bottom opening. We can approach the clogging transition by adjusting this opening. By placing the system between cross-polarizers, we can obtain high-speed video of this system during flow, and extract intensity signals that can be used to identify and quantify localized, otherwise indeterminate forces. We can simultaneously track individual particle motions, which can be used to identify shear transformation zones in the system. In this paper, we present our results thus far.
\end{abstract}

\section{Introduction}

The gravity-driven flow and clogging of grains through a silo has been studied for many years, and many empirical laws have been developed with some theoretical ideas offered [1-4], however the picture is not complete. In particular, we aim to understand the abrupt transition from flowing to clogging behavior. It has been thought that the clogging transition might be similar to the jamming transition, where some critical parameters (i.e. opening size) tell whether a clog will occur. With this picture, one also expects the requisite fluctuations (such as velocity fluctuations or dynamical heterogeneities) to appear in the system as the opening size approaches its critical value. However, recent work [2] on glass spheres has shown that while fluctuations are present, there is no variation in these fluctuations on approach to clogging, suggesting it is not a true transition. While this may be true for glass spheres, does this also hold for different particles? And more importantly, regardless of whether or not a true transition exists, how do fluctuations and intermittent structures (force chains, contact networks, shear zones) conspire to create a clog? To answer this question, we use photoelastic disks so that we may visualize the force chains in addition to tracking the particles' translations and contacts.

\section{Experimental Setup}

Our experimental setup consists of a quasi 2D silo filled with disks, a schematic is in Fig. 1A. The silo is $20 \mathrm{~cm}$ wide by $40 \mathrm{~cm}$ tall by $3.2 \mathrm{~mm}$ deep, made of clear plates screwed together, with the gap set by PTFE sheeting on

\footnotetext{
${ }^{\star}$ e-mail: thack22e@mtholyoke.ed
}

$\star \star$ e-mail: knordstr@mtholyoke.edu the left/right sides. The gap in the silo is such that we can fit a single layer of disks in the $20 \mathrm{~cm}$ x $40 \mathrm{~cm}$ area. Generally, the disks will not touch the front/back clear walls, the average disk will only touch other disks. We treat these walls with a (very) fine dusting of Teflon powder to ensure any incidental contact is low-friction. On the other hand, the disks on the side will touch the PTFE sidewalls on the left/right side of the apparatus.

The disks themselves are made of polyurethane. They are bidisperse (50:50 ratio) to suppress crystallization, with sizes of $3.9 \mathrm{~mm}$ and $6 \mathrm{~mm}$. The size of the large disks will be abbreviated with the symbol $D$ for the rest of this manuscript. The silo is filled with disks to a depth $h$. In all flow and clogging trials, we start from a fixed depth of 33 $\mathrm{cm}$. (A discussion of the height is included in Section 3). The Youngs modulus of the disks is $E \approx 3.5 \mathrm{MPa}$,

The silo bottom is made of PTFE inserts that can slide in and out to set opening sizes. The opening can be up to about $8 D$ in our current setup. For a fixed opening size, we insert a $3 \mathrm{D}$ printed plug into the gap. When we are ready to start a flow/clogging trial, we quickly remove this plug. The silo is attached to an external aluminum frame via an axle as depicted in Fig. 1B. The angle of the silo can be locked into place and is generally completely vertical. However, the silo can be removed or rotated easily to allow for quick resetting of the sample.

The silo is placed between crossed polarizers [4] and is backlit. This setup allows us to measure the photoelastic signal from the disks. Disks under stress will appear brighter than disks not under stress. Individual particle images can, in principle, be used to deduce the exact forces acting on each particle, but we have not set up sufficient spatial resolution to do this presently. We image the 


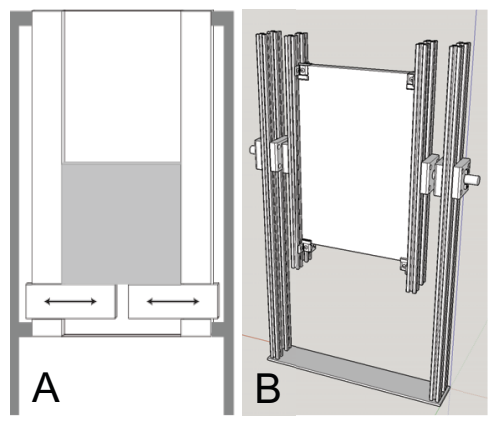

Figure 1. A) A schematic of the silo setup. PTFE inserts (double arrows) may be moved in or out to create openings of various sizes. B) The silo itself is coupled to an external frame with an axle. This allows the system to be rotated so the system may be reset, or completely inverted.

sample during flow/clogging at frame rates up to 10,000 fps using a Phantom CMOS camera. We do not need this high frame rate to capture the particle motion itself, but the force transmission (which appears as filaments of traveling brightness) between particles happens at much higher speeds. This points out another advantage to using these particles. Not only are they photoelastic, but since they are somewhat soft, the sound speed is much lower than an often-studied glass system, so we do have enough temporal resolution to capture this force transmission.

\section{System Characterization}

As this particular system has not been well-characterized before, we have started our investigation by seeing how this system behaves for two standard conditions. The first condition is static: do we see a characteristic pressure vs depth relationship? The second condition is in the freeflowing state: do we see flow curves that resemble other systems?

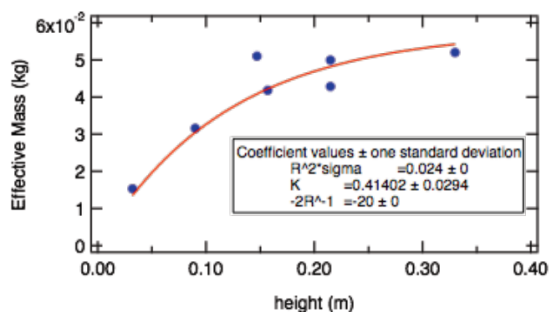

Figure 2. The effective mass of a system of 2D polyurethane disks as a function of height. We see that the Janssen effect is present in this system, and the data is consistent with Eq. 1.

To measure pressure vs depth in the sample, we remove the inserts that adjust the opening size and "plug" the bottom with a $3 \mathrm{D}$ printed rectangular insert that extends the width of the silo. This insert is then coupled to a force transducer. We fill the silo to various heights and measure the force at the bottom of the sample. We report this as "effective mass" in Fig. 2. The Janssen effect has widely been reported in granular systems, and is a pressure saturation vs depth, as the sidewalls can support some the weight of the pile. We present below a modified 2D expression based on an equation for a 3D system [5].

$$
M_{e f f}(h)=\frac{R^{2} \sigma}{K}\left(1-e^{-2 K h / R}\right)
$$

where $R$ is the half-width of the silo, the $h$ is the depth, $\sigma$ is the effective areal mass density. The parameter $K=$ $\mu P_{h} / P_{v}$, where $\mu$ is the coefficient of static friction and $P_{h} / P_{v}$ is the ratio of horizontal to vertical pressure, which should be of order 1 . Our data agrees with the functional form for Eq. 1. We find the value of $K$ to be about 0.41 , which coincides with our measurement of $\mu \approx 0.43$.

The second characterization of this system is in the free-flowing state. Generally, for these systems, freeflowing behavior is seen when the opening size is greater than about 5 times the particle size [1]. Calculation of the critical opening size involves experiments we will describe in a later section. However, even without this precise knowledge, it is simple to tell whether a particular opening size gives free-flow or not. We have looked at the flow rates vs time for several systems clearly in the freeflowing regime; we present example results from one trial in Fig. 3. To make this measurement, we fix an opening size and block the opening with a plug. We remove the plug and allow the particles to flow, and catch them in a pan. This pan is connected to a force transducer, which gives us a readout in real time. [It should be noted we have also made these same measurements using our video data, and we see consistent results.] We see some fluctuations and some transient behavior in the startup of flow, but overall the mass flow rate seems to be essentially constant. This is consistent with the Beverloo equation, an empirical equation developed for these systems, describing a constant flow rate. A 2D version is as follows [3]:

$$
W(D)=C \rho_{b} \sqrt{g}(D-k d)^{3 / 2}
$$

were $C$ is an empirically-determined discharge coefficient, generally between about 0.55 and $0.65, \rho_{b}$ is effective density of the material, $g$ is the acceleration due to gravity, and $k$ is the empirically-determined shape coefficient with a value generally between 1 and 3 . We see behavior consistent with this equation (Fig. 3).

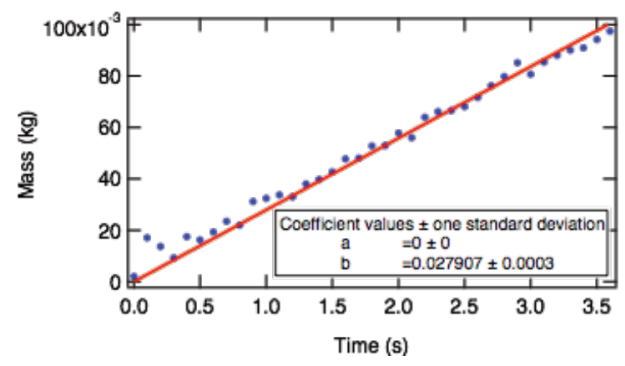

Figure 3. Mass discharged as a function of time for a freeflowing system. The slope obtained (the mass flow rate) is consistent with Eq. 2. 


\section{Bulk Avalanche Behavior}

We are interested in how clogs develop and intermittency in the bulk flow. In this section, we focus on opening sizes that are below the critical opening size. In this regime, a clog will form after some amount of time passes. We characterize these clogs in two ways: by looking at the size of the mass discharge before a clog, and by looking at the photoelastic signal of the entire sample.

We call the event preceding a clog an "avalanche." We characterize each avalanche by the total amount of mass discharged $(m)$. We start with the system filled, with a fixed opening size, and a plug blocking the opening. We remove the plug, and allow the sample to flow freely until a clog forms. To gather clogging statistics, we need tens to hundreds of trials at each opening size. We pour in particles lost during the last event and rotate the system on its side and shake it to reset the contacts. As a final measure to ensure a fresh run, we invert the entire sample; we have the same structure on the top and bottom to accommodate inserts and plugs. We create probability distributions of the discharged mass for each opening. We find that the data fit well to an exponential probability distribution: $P(m)=P_{0} e^{-m / m_{c}}$, where $P_{0}$ is a normalization constant. and $m_{c}$ is a fitting parameter with units of mass, which well call the "characteristic mass" of the opening size. We are able to this collapse the data in Fig. 4A. The fit to an exponential is consistent with the results in a $3 \mathrm{D}$ system of glass beads [2], and indicates that clogging can be considered a random Poisson process. By plotting characteristic mass vs opening size in Fig. 4B, we are able to see that $m_{c}$ looks to diverge as we approach a critical opening size, i.e. $m_{c} \sim\left(D-D_{c}\right)^{-\gamma}$. Further trials are currently needed to complete this piece of the story. With enough trials, we will get two pieces of information from this plot: the critical opening size $\left(D_{c}\right)$ and a critical exponent $(\gamma)$. We already know approximately what the critical opening size is so this will be a matter of increasing our precision. However, we are interested if our critical exponent $\gamma$ differs or agrees with those measured in [2].

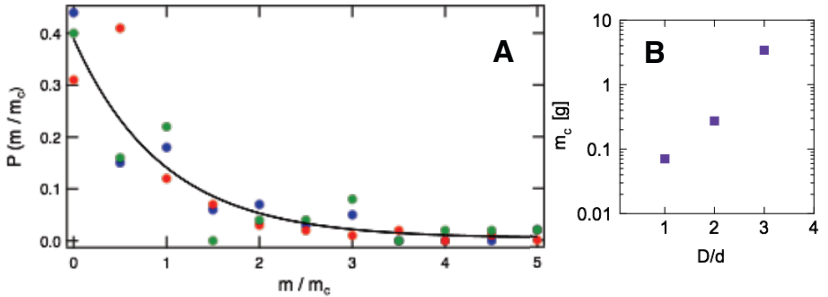

Figure 4. A) Probability distribution of mass discharged. Data are collapsed by rescaling $m$ by the characteristic mass $m_{c}$, obtained by fitting to a decaying exponential. B) $m_{c}$ obtained from fits in A) vs dimensionless opening size $(D / d)$.

By taking high speed video of clogging events, we can characterize the bulk flow in temporal detail. An example is shown in Fig. 5. In this video we have captured the photoelastic signal from the sample, and can also see individual particles clearly to confirm what is happening in

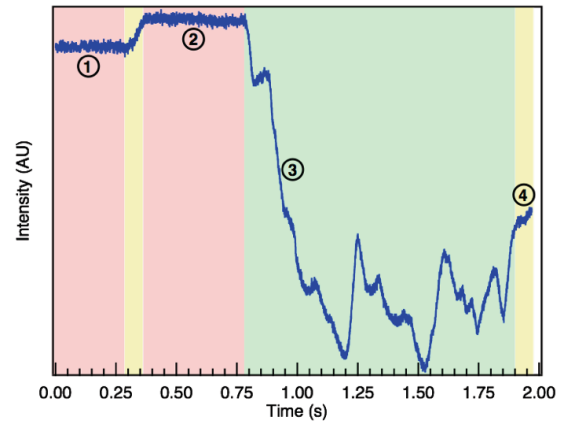

Figure 5. The intensity vs time of the photoelastic signal of the entire sample around a clogging event. 1) Before the plug is removed (pink, flat), then a brief period of avalanche (yellow, sloped) resulting in 2) a clog forming. The clog spontaneously breaks and a period of 3) free flow occurs, followed by 4) the formation of a new, permanent clog.

the sample. For this event, we have plotted the total intensity of a frame vs time. We have marked some key times with numbers in Fig. 5, and describe them here. 1) Before the plug is removed (pink, flat), then a brief period of avalanche (yellow, sloped) resulting in 2) a clog forming 3) free flow 4) A new, permanent clog forming. There are a few features of this event (and this method) to underscore. First, we see clogs as static intensity signals, and flow events as temporarily varying. This suggests a robust method to demarcate events in time as we have very high time resolution in the video signal. Secondly, there are three "static" states represented in this figure, all with different total intensities (stresses). This suggests a potential line of inquiry - are higher stress clogs more or less likely to spontaneously break? Third, we see intermittency in the flowing state, which echoes what we have seeing in the free-flowing state. Lastly, and most interestingly, we see a short-lived clog which breaks spontaneously. These temporary clogs are not captured in 3D (or 2D and low time resolution) studies, as the clog would be defined as the permanent clog (formed around $1.9 \mathrm{~s}$ for this data). Nonetheless these temporary clogs are very real and exist for finite amounts of time.

\section{Local Behavior}

We are interested in capturing local dynamics as clogs form. By taking photoelastic images at high frame rates, we are able to see local stresses within the sample at any given instant, and can also see forces being transmitted between particles. These force transmissions often occur much faster than the movement of the particles themselves. Of course, we are also able to track the particle positions. Briefly we use a circular Hough transform to find the particles, and MATLAB-based tracking algorithms to obtain their trajectories [6]. With the particle positions (and radii), we are also able to detect which particles are in contact with one another. In Fig. 6, we see these three complementary pieces of information (particle positions, force network, and contact network) plotted together. 


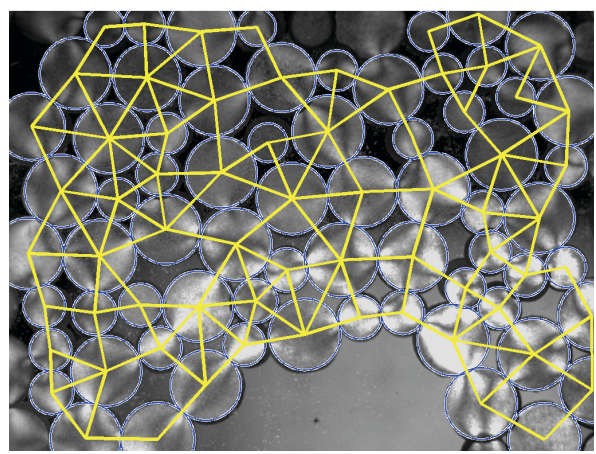

Figure 6. A zoomed-in image where an arch has formed over the opening, resulting in a clog. This composite image displays the photoelastic signal (grayscale intensity) which yields local force information, the particle positions (particles outlined in blue), and the contact network (yellow).

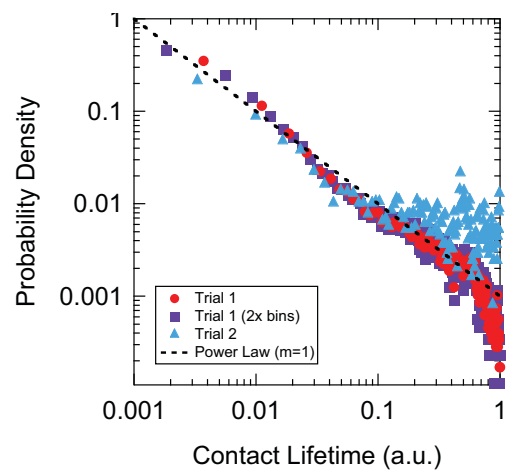

Figure 7. Probability density of contact lifetimes for two trials that $\operatorname{cog} D / d=4$. Two datasets are plotted for trial 1 (red circles and purple squares) this is to show the shape distribution does not depend on bin size. Trial 2 (blue triangles) clogs faster than 1.

Using the contact network data, we have made progress in characterizing the lifetime of contacts within the system. In Fig. 7, the probability density of contact lifetimes is plotted for two different trials which ultimately $\operatorname{clog}(D / d=4)$. A contact lifetime is defined simply as the time over which a single contact between two particles exists. In this figure, contact lifetimes are displayed for the whole sample ( $\sim 1000$ particles), not for just the zoomedin area near the arch. The contact lifetime statistics are gathered from the start of flow until the formation of a permanent clog. Both trials display a power law decay with an exponent close to 1 , as shown by the fit on the graph. In trial 2 , however, the clog forms sooner. We also see a larger number of longer-lived contacts. Speculatively, this might be the reason why trial 2 clogs sooner. We are actively pursuing this matter.

Lastly, we are interested in local avalanches within the system, that is, where and are major rearrangement events occurring? To do this, we quantify nonaffine (NA) motion within the system. NA motion can be the result of a variety of phenomena, including irreversible rearrangements or force chain breakage. Nonaffine motions point to deviations in the mechanical behavior of the granular material from a continuous medium. To measure NA motion, we use the quantity $D_{\min }^{2}$ as we have in previous work [6]: $D_{\text {min }, i}^{2}=\min \left\{\sum_{j}\left[\Delta \bar{d}_{i j}(t)-E_{i} \bar{d}_{i j}\right]\right\}^{2} . D_{m i n, i}^{2}$ quantifies the NA motion of $j$ particles in the neighborhood around a given particle $i$ after removing the averaged linear response to the strain, given by tensor $E_{i}$; a larger $D_{\text {min }}^{2}$ indicates more NA motion. The vector $\bar{d}_{i j}$ is the relative position of $i$ and $j, \Delta \bar{d}_{i j}$ is the relative displacement. Fig. 8 shows a map of $D_{\text {min }}^{2}$ for a particular time in the free-flowing system. We see that there are several regions of high $D_{\min }^{2}$, but these do not always correspond to high-shear regions near the opening. By connecting these measurements with the force and contact networks we hope to elucidate why this is. Are these rearrangements far from the opening important? Indeed, we generally see that while the overall flow rate can be fit well to the (smooth) Beverloo equation, we can see by eye (and with the photoelastic signal) that the bulk motion is quite jerky. Presumably this influences clog formation when the system is in this regime.

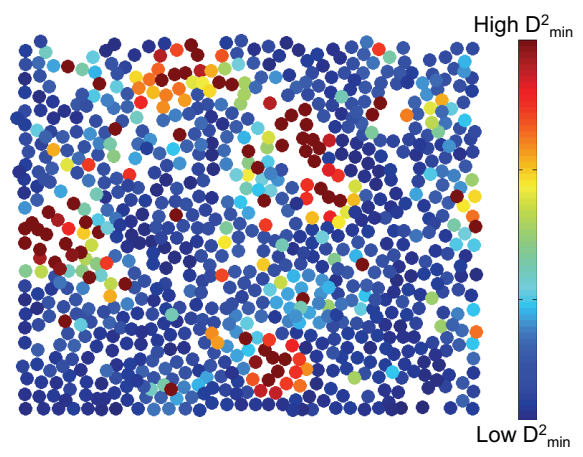

Figure 8. A plot of $D_{\min , i}^{2}$ in a freely-flowing system partway through the outflow. The opening is about $7 \mathrm{D}$ and is bottom center in this plot. Warm colors denote "hotspots" of rearrangements and cool colors denote areas where the particles move according to the average flow.

\section{Acknowledgements}

The authors thank Kathryn Morrison, Len McEachern and Thomas Liimatainen. This work is partially funded by ACS award PRF 56888-UN19.

\section{References}

[1] K. To. Physical Review E, 71, 060301 (2005).

[2] C. C. Thomas and D. J. Durian, Physical Review E, 87, 052201 (2013).

[3] C. Mankoc, A. Janda, R. Arevalo, J. M. Pastor, I. Zuriguel, A. Garcimartin, and D. Maza, Granular Matter, 9, 407 (2007).

[4] J. Tang and R. P. Behringer, Chaos, 21, 041107 (2011).

[5] R. Back. Granular Matter, 13, 723 (2011).

[6] K. N. Nordstrom, E. Lim, M. Harrington, and W. Losert Physical Review Letters, 112, 228002 (2014). 